\title{
FORMACIÓN DE PROFESORES INDÍGENAS Y CONDICIONES PARA LA APROPIACIÓN DE LAS TECNOLOGÍAS DE LA INFORMACIÓN Y LA COMUNICACIÓN
}

\author{
EDUCATING NATIVE TEACHERS AND CONDITIONS FOR \\ THE APPROPRIATION OF ENCICLOMEDIA
}

Amelia Molina García Alma Epifania López Quiterio

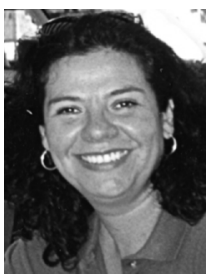

Doctora en Ciencias de la Educación, Universidad Autónoma del Estado de Hidalgo (UAEH). Maestra en Investigación y Desarrollo de la Educación, Universidad Iberoamericana. Licenciada en Pedagogía, Universidad Nacional Autónoma de México (UNAM). Desde hace más de 20 años se dedica al campo de la educación, desde nivel básico hasta superior. Actualmente es profesora investigadora de la $\mathrm{UAEH}$ y ha participado en diversos proyectos de investigación. Pertenece al Sistema Nacional de Investigadores en el nivel I.

Correo electrónico: [meluxmolina@hotmail.com].
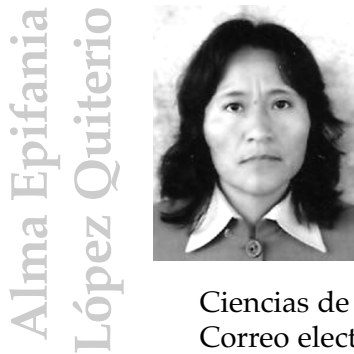

Maestría en Ciencias de la Educación, Universidad Autónoma del Estado de Hidalgo (UAEH). Profesora de Educación Primaria, UAEH. Posee una amplia experiencia en las diversas modalidades de educación básica y ha participado en varios proyectos de investigación. Actualmente estudia el Doctorado en Ciencias de la Educación, UAEH.

Correo electrónico: [aelopezq@hotmail.com].

\section{RESUMEN}

Este trabajo presenta los resultados parciales de una investigación de tipo mixto, en donde se analiza la relación entre formación docente y práctica educativa de profesores de educación indígena, particularmente con el uso de las Tecnologías de la Información y la 
Comunicación (TIC). Los resultados evidencian que en la formación inicial de los docentes se da poca importancia a la enseñanza sobre las TIC. Los cursos de actualización en materia de TIC son muchos y variados, pero no atienden a las necesidades de formación que los profesores requieren, pues no se basan en un diagnóstico sólido y mucho menos están enfocados a las condiciones particulares de los contextos indígenas. No obstante, se generan estrategias para una formación de calidad, con herramientas para la vida. Para desarrollar esta investigación, nos ocupamos de aspectos que articulan las demandas de la Sociedad del Conocimiento y las condiciones de las comunidades indígenas. Exponemos, conceptualmente, lo que se entiende por «sociedad del conocimiento» y lo que implica trabajar desde este paradigma en el proceso de «apropiación» en la generación de conocimiento desde los ámbitos indígenas.

Palabras clave: tecnologías de información y comunicación en la educación; formación inicial docente; contextos indígenas.

\begin{abstract}
This paper presents the results of a research of mixed type, which analyzes the relationship between teacher training and educational practice of teachers of indigenous education, particularly with the use of Enciclomedia. The results show that in the initial training of teachers, it is given little attention to the teaching about Information Technology and Communication (ICT). The updating courses in ICT are many and varied, but do not attend the training needs that teachers require, they are not based on sound diagnosis and are not focused on the particular conditions of the indigenous contexts. However, strategies for quality education are generated, with tools for life. To develop this research, we deal with issues that articulate the demands of «knowledge society» and the conditions of indigenous communities. We describe conceptually what is meant by Knowledge Society and the implications of working from this paradigm in the process of «appropriation» in the generation of knowledge at indigenous areas.
\end{abstract}

Key words: Information Technology and Communication on education; initial teachers' training; indigenous context. 


\section{PRESENTACIÓN}

La incorporación de Tecnologías de la Información y la Comunicación (TIC) al campo de la educación, ha sido relativamente reciente, como respuesta a las tendencias internacionales para ingresar a la Sociedad de la Información y del Conocimiento. En México, además de algunas otras estrategias, se implementó el programa Enciclomedia, con el propósito de contribuir a la mejora de la calidad en la educación primaria, por medio de la experimentación e interacción de los contenidos educativos incorporados en este programa. Si bien las investigaciones relacionadas con Enciclomedia plantean perspectivas (pedagógicas, didácticas, económicas, políticas de uso e implementación y apropiación social, entre otras), los contenidos de la investigación a la que hacemos referencia en este artículo, se dirigen al análisis de las condiciones que presentan los profesores de primaria para trabajar con las TIC y, en su caso, apropiarse de Enciclomedia como recurso de apoyo en su práctica educativa en contextos de primaria indígena. Por tanto, los propósitos del estudio son: caracterizar la formación inicial y el desarrollo profesional del docente de educación indígena; analizar el uso y la apropiación tecnológica que realiza el docente en el aula en los contextos indígenas, para comprender el significado que otorgan a las TIC.

\section{APROXIMACIÓN CONCEPTUAL AL OBJETO DE ESTUDIO}

Hoy, la difusión de la información se ha vuelto más rápida debido a las TIC; la velocidad de procesamiento de la información y las formas de almacenamiento, permiten la trasmisión en mayor cantidad y en menor tiempo y espacio. Se constituyen, así, sociedades de la información, modificando las formas de percibir lo local y lo regional, lo nacional y lo mundial.

La Sociedad de la Información puede entenderse como la expresión de las realidades y las capacidades de los medios de comunicación para transmitir información gracias a la digitalización (Trejo, 2001: 4). $\mathrm{Al}$ enfatizar el papel de la información en la sociedad, Castells (2008: 46-47) la denomina «sociedad informacional», refiriéndose a la información como comunicación del conocimiento. Es decir, reconoce que la información es solo un instrumento para acceder al conocimiento. 
Aprovechar las TIC en el desarrollo de la capacidad de procesamiento de la información y la habilidad para crear redes en beneficio mutuo que contribuyan a la mejora del nivel de vida, constituye un reto. Sobre todo porque no todas las personas tienen acceso a la información, pues los procesos que expandieron las TIC, se vincularon con el proceso de globalización económica que planteó la aplicación de los avances tecnológicos como detonante para el desarrollo económico al punto que, en la Declaración de Florianópolis, en Brasil, los gobiernos de América Latina y el Caribe anunciaron su aspiración

[...] de llegar al año 2005, integrados como miembros plenos de la Sociedad de la información con eficiencia, equidad y sustentabilidad, en el marco de la economía global basada en el conocimiento (Florianópolis, 2000: 1).

De igual forma, reconocieron el riesgo de aumentar las brechas sociales al interior de la sociedad, de crear nuevas formas de exclusión, de expandir las condiciones para la globalización y de incrementar la distancia entre los países, pero también al interior de ellos. Se reforzaría, así, la acumulación de conocimientos y aplicaciones científicas en beneficio de los países más desarrollados, alejando progresivamente las posibilidades de acceso a esta tecnología por parte de las minorías, particularmente con las poblaciones más vulnerables, entre ellas las comunidades indígenas (Adell, 1997: 22; Álamo, 2004: 1).

El acceso y aprovechamiento de las TIC, como medio de crecimiento económico, sociocultural y de participación política, está limitado o se encuentra en situación de rezago para las comunidades indígenas. Se identifica una brecha digital que es necesario revertir. Algunos investigadores, intelectuales y organismos internacionales, preocupados por las poblaciones indígenas, buscan lograr una verdadera sociedad de la información global, incluyente y democrática; luchando por tener su propio ciberespacio y gozar del uso de las TIC, a través del impulso de distintos foros y congresos que permiten revalorar a las culturas indígenas y considerar a las TIC como una valiosa oportunidad para trascender del nivel local al nivel nacional e internacional en distintos ámbitos. Entre ellos, cabe mencionar el informe final del II Encuentro sobre Conectividad y Poblaciones Indígenas en Ottawa y la Declaración del Encuentro Indígena Interamericano Preparatorio, para la Cumbre Mundial en 
Brasilia (Sandoval y Mota, 2005: 7); la Cumbre Mundial de la Sociedad de la Información (CMSI) de Ginebra 2003 y Túnez 2005 (CMSI, 2005: 1).

El acceso a la Sociedad de la información puede no ser suficiente para contribuir al desarrollo de las sociedades indígenas ya que, para hacer frente al desafío de una Sociedad Mundial de la Información (SMI), es importante que los procesos de aproximación se trabajen desde la noción de aprendizaje con una perspectiva crítica. Es decir, es fundamental considerar que se debe aprender a aprender:

[...] aprender a reflexionar, dudar, adaptarse con la mayor rapidez posible y saber cuestionar el legado cultural propio, respetando los consensos (UNESCO, 2005: 66).

Es preciso, entonces, que las sociedades indígenas participen en la Sociedad de la Información como generadoras y gestoras de los contenidos que muestren su riqueza cultural, su cosmovisión, su pensamiento, su sensibilidad y su creatividad (Medellín y Huerta, 2006: 5). De igual forma, es conveniente señalar que la SMI cobrará sentido si se convierte en un medio para construir Sociedades del Conocimiento como fuente de desarrollo para todos los países.

A partir de la idea de que la información no es conocimiento, es pertinente diferenciar estos conceptos. Para Casas y Dettmer:

[...] la información toma la forma de un conjunto de datos estructurados $\mathrm{y}$ formateados que permanecen pasivos hasta que son usados por quienes poseen el conocimiento necesario para interpretarlos y procesarlos (2008: 28).

En tanto que la reproducción del conocimiento es más compleja, debido a que «la capacidad cognitiva no es fácil de articularse explícitamente o de transferirla a otros» (David y Foray, cit. en Casas y Dettmer, 2008: 29), sobre todo cuando el conocimiento no es explícito.

De acuerdo con Montushi (2001: 12-13), existen formas de almacenar el conocimiento: explícita o tácitamente. El conocimiento tácito es aquel que está incorporado en la persona sin ser accesible permanentemente a la conciencia, pero que el individuo utiliza cuando las 
circunstancias lo requieren; dicho conocimiento es subjetivo y está basado en la experiencia. En muchas circunstancias, este conocimiento es específico a un contexto, por lo cual es difícil - a veces incluso imposible- expresarlo en palabras, oraciones, números o fórmulas. Abarca también creencias, imágenes, intuición y modelos mentales, así como habilidades técnicas y artesanales. Puede ser denominado «conocimiento individual implícito», «conocimiento incorporado»o el «conocer cómo» (know-how).

En contraposición está el conocimiento explícito, el cual es el conocimiento objetivo y racional; suele identificarse como conocimiento codificado, ya que se expresa de manera formal y es transmitido a otros de modo igualmente sencillo independientemente del contexto. Bajo esta lógica, el conocimiento explícito puede ser medido con relativa facilidad, no así el conocimiento tácito y el know-how práctico, que presentan mayores dificultades.

De acuerdo con Foray y Lundvall (cit. en Casas y Dettmer, 2008: 30), existen distintos tipos de conocimientos, que aluden a la manera en que se aprenden o interiorizan:

1) Know-what (saber qué): conocimiento acerca de «hechos», es decir, «información».

2) Know-why (saber por qué): refiere al conocimiento científico que se produce generalmente en organizaciones especializadas.

3) Know-how (saber cómo): identificado como el conocimiento que soporta la habilidad y/o capacidad de hacer algo.

4) Know-who (saber quién): este conocimiento implica la mezcla de diferentes tipos de habilidades, incluidas las que posibilitan las relaciones sociales, que permiten tener acceso al experto y usar su conocimiento eficientemente. Involucra el poder identificar o tener la información acerca de quién sabe qué, y quién sabe cómo hacer qué.

De acuerdo con la Organización para la Cooperación y el Desarrollo Económico (OCDE), los dos primeros son conocimientos codificados o explícitos, mientras que los dos últimos, se vinculan con el conocimiento tácito o implícito. Por tanto, las TIC estarían dando respuesta a las necesidades de manejo de los conocimientos 
codificados, es decir, los conocimientos del tipo «saber qué» y el «saber por qué» (Cervantes, 2006: 93-94; Montushi, 2001: 10).

Es importante la distinción entre los tipos de conocimiento, pues con ello se alude al modo en que los individuos se los apropian para generar o producir nuevos conocimientos. En este marco, la educación cobra especial importancia, ya que los retos para participar en una Sociedad del Conocimiento, que dispone de grandes cantidades de información en la red mundial, demanda una nueva manera de comprender lo social, centrada en: la capacidad de crear y compartir conocimiento (Carrión, 2007: 12); la búsqueda de la libertad de expresión; la calidad y la diversificación de contenidos; la diversidad cultural y lingüística; el derecho de comunicar, y el dominio de competencias cognitivas y reflexivas a partir de las tecnologías digitales.

Sin embargo, aun cuando se plantea la distinción entre los tipos de conocimiento que entran en las TIC y lo que demanda para su producción y aprendizaje, nos preguntamos: ¿los conocimientos que producen las poblaciones indígenas, (implícitos o tácitos) y las formas de transmisión, se encuentran en desventaja? Como señala Barahona (cit. en Gómez, 2006: 9), los conocimientos indígenas forman parte de los conocimientos tácitos y deben comprenderse como la suma de ideas y percepciones de lo que se considera como sistema cognitivo indígena; su almacenamiento y transmisión son básicamente nemotécnicos y orales. Además de que este conocimiento no es catalogado como «científico», pues no se produce institucionalmente.

Ante tal circunstancia, se puede señalar que los conocimientos indígenas se someten a una doble lucha: por un lado, el reconocimiento de sus saberes y, por otro, la posibilidad de utilizar otros medios o nuevas estrategias para su transmisión. En estas condiciones, es pertinente definir que los conocimientos indígenas, de acuerdo con Gurvich (1992 cit. en Gómez, 2006: 8), «son diferentes modos de expresión, comunicación y difusión del saber siempre en correlaciones funcionales con los sistemas colectivos de emisores y receptores»; y que no están anclados en el pasado, ni en el atraso, pues estos conocimientos tienen una vigencia amplia. De esta manera se observa la pervivencia de las culturas indígenas, que han sido capaces de adaptarse a las nuevas circunstancias. 
Hablar de conocimientos indígenas (Gómez, 2006: 9), implica reconocer que ellos no son sujetos enmarcados en estereotipos creados y manejados por los medios de comunicación (visión folclórica), sino que son sujetos de información y comunicación; que pertenecen a culturas milenarias; que tienen presencia, en pleno siglo XXI; que se derivan de la participación y organización en comunidades y organizaciones productivas que han sido capaces de adaptarse a esta modernidad líquida —en el sentido de Bauman (2007: 28)—. Por tanto, han logrado que pervivan sus conocimientos a lo largo de siglos y pueden constituir nuevas esperanzas de solución a muchos de los problemas que la sociedad enfrenta con relación, por ejemplo, al cuidado del medio ambiente.

Con la llegada de las TIC, algunas culturas indígenas se han apropiado de estas para apoyar la escritura en sus lenguas, creando sus propios grafismos, conservando los sonidos y conceptos ante el alfabeto latino; y por otro lado, también han logrado organizarse para oponerse a la transnacionalización y privatización de sus tierras, argumentando el impacto devastador que dichas acciones tendrían en el medio ambiente.

Los conocimientos indígenas son distintos a los occidentales. Estos últimos, pueden alienarse de su creador, industrializarse y aplicarse fuera del contexto territorial, social y cultural en que fueron creados y se convierten, apresuradamente, en mercancía. No así los conocimientos indígenas, cuya riqueza estriba en su aplicabilidad dentro del contexto social y cultural donde fueron producidos (Gómez, 2006: 9). Sin embargo, deben aprovecharse todas las aportaciones en un marco de respeto y colaboración para lograr mejores niveles en la calidad de vida, como lo señala la UNESCO:

Las sociedades del conocimiento solo podrán evitar los escollos de la homogeneización cultural y del relativismo cultural, haciendo hincapié en la necesidad de los valores compartidos que permitan construir un auténtico pluralismo (2005: 168).

Bajo este marco, y con fines analíticos para el desarrollo del estudio, tomamos como referentes los tres niveles fundamentales que forman parte del proceso de apropiación para una Sociedad del 
Conocimiento, los cuales son: acceso, capacitación y apropiación (Cobo, 2007: 21-27; 2005: 47-48).

Acceso: Contar con los medios y la infraestructura tecnológica que garanticen la accesibilidad y conexión a Internet y otras redes.

Adopción: Determinar el proceso de aprendizaje en el uso de las TIC, con el dominio (menor o experto) y el aprovechamiento funcional de las TIC. Esta etapa implica romper con barreras como resistencias de usuarios educados en un entorno analógico o no contar con los incentivos necesarios para motivar a los potenciales usuarios de estas herramientas. Se determina por el desarrollo de las habilidades informáticas y las capacidades de acceder a la información requerida por el usuario.

Apropiación: Emplear las TIC para estimular el aprendizaje y desarrollar habilidades que contribuyan a la creación de nuevo conocimiento. Su característica central es que los usuarios son capaces de incorporar nuevas formas de emplear la información, creando y compartiendo el conocimiento de manera horizontal y distribuida. De lo contrario, las tecnologías serán subutilizadas y no se logrará la resignificación necesaria para avanzar hacia la formación de ciudadanos para una Sociedad del Conocimiento.

En síntesis, la apropiación refiere a la posibilidad de emplear las TIC de manera efectiva: cuando se reconocen sus limitaciones y posibilidades en cada contexto; cuando los individuos las adecuan a su propia forma de concebir el mundo y de intervenirlo; cuando comprenden los códigos y significados de las TIC (es decir, no están separados de la cultura, sino que son forma de expresión de la misma) (Cabrera, 2006: 4; Sagástegui, 2006: 2; Morales et al., 2004: 4).

El uso y apropiación de las TIC en los procesos de enseñanza, abre nuevas posibilidades y plantea nuevas exigencias a los sistemas escolares para diseñar el aprendizaje del siglo XXI, situación que demanda transformar el qué y el cómo se aprende, así como el funcionamiento de las instituciones educativas. Por ello, como mencionamos al inicio de este trabajo, la pretensión es revisar qué sucede con la incorporación de las TIC al proceso de enseñanza, en especial en contextos indígenas. 


\section{METODOLOGÍA}

El estudio fue de carácter exploratorio (Garza, 1988), basado en una metodología mixta de investigación, que posibilitó una aproximación cuantitativa y cualitativa del objeto de estudio. Para la primera, se realizó una encuesta para conocer el nivel de formación de los docentes participantes en la investigación; se aplicó un cuestionario y los datos obtenidos se procesaron mediante el Programa de Estadística para Ciencias Sociales (SPSS). Si bien parte de los indicadores fueron tomados de un instrumento ${ }^{1}$ validado y probado con poblaciones similares, para este estudio se realizó una adaptación para profesores de educación indígena, en la que se consideraron cinco de los siete indicadores propuestos:

I. Condiciones socioeconómicas. Se refieren al tipo de vivienda en que se habita, el número de personas que aportan ingresos al gasto familiar y el nivel máximo de estudios de los padres.

II. Formación académica. Incluye información relativa a los estudios realizados (Normal básica, Normal superior y otras licenciaturas), previos al ejercicio docente.

III. Percepciones sobre formación inicial y actualización. Las valoraciones de los profesores sobre su formación inicial y la actualización, relacionadas con su práctica docente, así como su experiencia y sus necesidades, se incluyeron en este apartado.

IV. Trabajo docente y aula. En este rubro se recogen las apreciaciones de los profesores sobre algunos problemas y sobre la modalidad que asume su práctica docente -específicamente lo relacionado con educación indígena-, así como los elementos de que disponen para desplegarla (manejo de teorías y metodologías, conocimientos sobre los alumnos, diseño curricular y evaluación, entre otros).

V. Innovación educativa. Alude a las características de habilitación de materiales y equipo tecnológico del que disponen las escuelas, así como a la valoración sobre el dominio de los profesores sobre estas, en especial de la Enciclomedia.

Para la construcción del instrumento se tomó como base la Encuesta sobre Necesidades de Formación y Prácticas Institucionales de Profesores de Educación Secundaria, Ducoing y Miranda, SEP, 2004. 
Antes de la aplicación definitiva, el cuestionario se piloteó en dos ocasiones con una muestra representativa en una zona escolar con características similares a la zona objeto de estudio, para verificar su validez y confiabilidad.

\section{ELECCIÓN DE LA MUESTRA}

La población participante en la investigación fue elegida considerando los criterios de muestra tipo intencional, en los que se consideró la disposición de las autoridades oficiales para facilitar el estudio; que los profesores estuvieran ubicados en contextos indígenas, independientemente de que sus escuelas contaran o no con equipo Enciclomedia. La zona escolar elegida se localiza en el municipio de Chilcuautla, en el Estado de Hidalgo. Son comunidades que preservan su cultura originaria, razón que posibilitó el alcance del objetivo de investigación: conocer cómo los maestros emplean las TIC y las adaptan a las necesidades particulares de los niños indígenas.

Para caracterizar a los docentes frente a grupo de dicha zona, se aplicó el cuestionario a 41 profesores de un total de 46, teniendo una pérdida de $10.86 \%$ que no proporcionó respuesta. Los docentes encuestados se distribuyen en 11 escuelas de la zona.

En la elección de la muestra cualitativa (dos escuelas) se consideraron los siguientes criterios: poseer equipo Enciclomedia y ser de organización completa (una de ellas, con población estudiantil que emplea como lengua materna el idioma indígena y otra cuya lengua materna es el español, ambas ubicadas en comunidades indígenas).

\section{APLICACIÓN}

Los datos generales de los profesores de la zona escolar elegida, se obtuvieron mediante el cuestionario. Posteriormente - y con la finalidad de recuperar información sobre los procesos de desarrollo y desempeño docente en las escuelas y grados seleccionados- se realizaron registros mediante la técnica de observación participante (Sánchez, 2008: 97-131). De la misma manera, se aplicaron entrevistas semiestructuradas a los docentes observados, con el objetivo de 
conocer su opinión sobre el manejo de Enciclomedia como recurso en la práctica educativa. También se realizaron entrevistas etnográficas con algunos otros agentes que, en el momento de la observación participante, se encontraban inmersos en los procesos educativos.

\section{PROCEDIMIENTO ANALÍTICO}

El análisis de la información se llevó a cabo con la técnica de triangulación, en la que se consideran los datos (respuestas al cuestionario) sobre formación docente en TIC y las entrevistas y los registros de observación en los salones de clase. En este ejercicio, los ejes ordenadores fueron: los docentes, los procesos pedagógicos y las condiciones institucionales. Mediante ellos se identificó el tipo de formación que poseía el docente sobre el uso de TIC; con la observación de la práctica educativa se contó con elementos para contrastar cómo ese saber que poseía o no el docente, influía en su práctica; con el análisis de las entrevistas se contrastaron y complementaron las situaciones observadas, otorgándoles los significados que los propios sujetos investigados les asignaron. Es importante mencionar que este hecho sirvió para dar cuenta de la realidad estudiada, a partir de la contrastación de la información, evitando sesgos que afectaran su validez.

\section{ANÁLISIS DE RESULTADOS Y CONCLUSIONES}

Uno de los ejes principales de las Sociedades del Conocimiento, es que los sujetos construyan conocimientos para responder a las demandas crecientes de un mundo cambiante, en donde la simple transmisión de información no basta para que los ciudadanos enfrenten con dinamismo los embates del futuro. Es indispensable un aprendizaje permanente; un aprendizaje a lo largo de la vida, que permita enfrentar las nuevas demandas laborales y sociales en un mundo cada vez más diversificado; donde no solo se exige determinado capital humano, sino la capacidad de relacionarse con los otros en términos de respeto y colaboración; donde lo afectivo cobra importancia, dada la situación de conflicto, de problemas étnicos, de exclusión y de discriminación, entre otros. 
Entender cómo el docente de educación indígena enfrenta estos desafíos, particularmente con la incorporación de las TIC en los espacios educativos, implicó conocer sus opiniones, el trabajo realizado en las aulas frente alumnos culturalmente diversos, con lenguajes distintos, donde el proceso de socialización primaria (Berger y Luckman, 1997: 164-173) también es diverso. Así, a partir de la información recopilada, se concluyó lo siguiente:

1. La formación inicial de los docentes indígenas no contempla el empleo de las TIC en la educación; este solo se realiza de manera instrumental y con una o hasta dos materias al respecto. Por tanto, no cuentan con bases que les permitan adquirir las competencias necesarias para integrar curricularmente las TIC, tanto en la enseñanza como en el aprendizaje permanente del profesorado.

2. La formación por sí sola no resuelve el problema de la incorporación o no de las TIC a la escuela; hace falta una reestructuración de la práctica educativa en el subsistema de educación indígena, pues dichas políticas educativas promueven acciones uniformes, sin considerar las particularidades y necesidades de cada escuela, docente y alumnos.

3. Se diseñan cursos de capacitación - muchos y variados- en torno al empleo de las TIC para apoyar la enseñanza, pero estos no llegan a los destinatarios finales, lo cual sugiere una falta de seguimiento y evaluación de los mismos.

4. La formación en las TIC debe ir más allá del aprendizaje técnico, debe también facilitarse el acceso regular a la computadora, tanto en la escuela como en la casa, para que los docentes exploren y diversifiquen su incorporación en la enseñanza.

5. En gran parte de los casos, la Enciclomedia ha sido el único recurso de los docentes para acercarse a las TIC, pero no cuentan con el tiempo suficiente para explorarla; en el caso de los nuevos materiales curriculares, se sugieren páginas de consulta pero tampoco pueden acceder a ellas, porque la mayoría de las escuelas no tiene el servicio permanente de Internet. 
6. No se cuenta con documentos o materiales que permitan entrar al programa Enciclomedia en otro lugar que no sea la escuela ya que, de acuerdo con la información contextual sobre las características y bienes materiales de las comunidades de estudio, es posible desarrollar este tipo de actividades en espacios ajenos a la escuela.

7. El proceso de apropiación de las TIC, por parte de los docentes, no se logra porque muchos de ellos apenas se inician en el manejo técnico de las TIC, sobre todo porque apropiarse significa: hacer suyo y adecuar la herramienta a sus necesidades y situaciones particulares.

8. Se cree que el docente debe únicamente capacitarse; no se contempla su formación inicial, ni se realiza un diagnóstico que dé cuenta de las competencias que posee o las que le son necesarias para comprender los conceptos y teorías que plantean las reformas; es decir, de acuerdo con lo que se encontró, falta apropiación de las TIC.

9. Se exige al docente que aprenda a enseñar de forma diferente a como él fue enseñado; sin embargo, ni siquiera los formadores han sido capacitados para ello, por tanto, se siguen reproduciendo las viejas prácticas.

10. En el caso de las comunidades indígenas, en muchas ocasiones, el único recurso para obtener información son los libros de texto que otorga la SEP; con ello, Enciclomedia constituye una valiosa herramienta que provee información, pero deben proyectarse otras formas de acceso a los recursos del programa, para que el docente planee sus clases y diseñe estrategias que permitan la apropiación y el desarrollo de conocimientos por parte de los alumnos.

11. El programa Enciclomedia señala que se han realizado, en video, una serie de traducciones de contenidos de materiales en lenguas indígenas; sin embargo, durante los períodos de observación, estos recursos no se emplearon. Aunque los equipos cuentan con la versión 2.0 - que permite la accesibilidad e incorporación de contenidos en distintas lenguas-, los docentes no conocen el funcionamiento de dicho reproductor y los materiales en lenguas indígenas son limitados. 
12. La educación indígena se reduce a lo lingüístico, aspecto que se confirma con el establecimiento de parámetros curriculares para la asignatura de lengua indígena, la cual propone contenidos que deben tratarse en el área del lenguaje, bajo el supuesto de que todos los niños que asisten a las escuelas indígenas tienen como lengua materna un idioma indígena (situación que de acuerdo con lo observado no es así). Esto, en cierta forma, es atribuible a las políticas educativas anteriores, que usualmente exigían la erradicación de las lenguas indígenas, o su empleo solo para la castellanización. En este caso, son pocas las comunidades en donde los alumnos emplean, como lengua materna, un idioma indígena.

13. En algunas escuelas, lo que se realiza es un programa de recuperación de dichas lenguas. Además, de acuerdo con los lineamientos para la Educación Intercultural Bilingüe, se pretenden recuperar también los saberes locales. Para ello se han propuesto algunos materiales, como: «La educación intercultural bilingüe», que generalmente cumple una función simbólica, ya que aunque se cuenta con ellos, los docentes observados nunca los usaron.

14. Los docentes de educación indígena de esta zona, no poseen la formación pedagógica para integrar curricularmente a las TIC en su práctica educativa, ya que iniciaron su labor educativa tan solo con secundaria, bachillerato o, en el mejor de los casos, con nivelación pedagógica como formación inicial.

15. Actualmente, aunque poseen una licenciatura - aspecto que refleja a un docente preocupado por continuar con su desarrollo profesional-, no están en posibilidad de ofrecer altos resultados educativos, puesto que las licenciaturas cursadas no son acordes con el perfil, o en los casos donde sí lo es, no han reflejado esta formación al atender a la población indígena.

16. Las políticas educativas plantean subsanar estas carencias con programas de capacitación, pero no responden al contexto.

17. Las TIC pueden ser importantes aliadas del aprendizaje si se reconoce su potencial educativo, tanto dentro como fuera del sistema escolar. Existe una necesidad de que distintos proyectos en las TIC sean aprovechados para este fin y puedan ser articulados. 
18. En el caso de las escuelas observadas, las comunidades tienen proyectos - como los Centros Comunitarios de Aprendizaje o el albergue escolar- que proporcionan acceso a Internet y programas que pueden coadyuvar a la mejora de la calidad educativa, pero no existe una coordinación de esfuerzos.

19. Se pueden diseñar proyectos educativos por los propios docentes - que conlleven al diseño de materiales para contribuir al desarrollo efectivo de una educación indígena, que contemple no solo la parte lingüística, sino la recuperación de los saberes indígenas- para favorecer aprendizajes significativos en los alumnos y para mejorar la calidad de vida de las propias comunidades, involucrando a los padres de familia en esta tarea, pero se requiere de una adecuada labor de gestión escolar.

20. No existe un trabajo integrado en las escuelas, porque se prioriza el trabajo individual de los docentes. Se requiere de comunidades de aprendizaje que permitan construir proyectos educativos con base en las necesidades propias de cada escuela, en el uso adecuado del tiempo y del conocimiento tácito que cada docente posee.

21. Existen riesgos enormes entre los planteamientos de una educación diversificada, porque en el afán de respetar las particularidades de cada grupo - en este caso indígena-, puede provocarse la ausencia de otras competencias que dejan al alumno indefenso ante las nuevas exigencias de una Sociedad del Conocimiento. Por ejemplo, en el caso de la asignatura estatal, la lengua adicional: los niños que asisten a escuelas primarias generales, sean indígenas o no, pueden aprender una lengua extranjera, pero los niños que asisten a escuelas indígenas prescriptivamente, deben aprender una lengua indígena dada la particularidad de estas, lo que estaría limitando el desarrollo de la competencia comunicativa en el denominado «idioma universal» (el inglés, que se percibe como una herramienta para la movilidad de jóvenes y adultos hacia los estados y países del norte).

22. El problema no es si deben aprender una lengua extranjera o no, sino qué oportunidades se les brindan para desempeñarse en un mundo en donde se requiere dominar, por lo menos, el idioma 
inglés. Como un acierto, pudimos observar que en esta zona, la supervisión escolar tiene clara la necesidad de aprendizaje del idioma inglés (condición migrante hacia los Estados Unidos), ya que se promueve tanto el aprendizaje de la lengua indígena como la del inglés, aprovechando el recurso Enciclomedia.

23. Finalmente, señalamos: no se trata solo de dotar de infraestructura a las escuelas para afirmar que la educación es equitativa, sino de proporcionar las condiciones de equidad para que todos los niños -independientemente de su condición étnica- desarrollen las competencias indispensables para afrontar los retos de una Sociedad del Conocimiento. Los reformadores plantean la importancia de formar a los alumnos para la vida, pero no se generan las condiciones (espacios para hacer) en que los docentes y alumnos involucren la movilización de saberes en situaciones comunes de la vida diaria y en escenarios complejos que los lleven a reestructurar las competencias desarrolladas.

\section{REFERENCIAS BIBLIOGRÁFICAS}

ADELL, Jordi, «Tendencias en educación en la sociedad de las tecnologías de la información», Revista Electrónica de Tecnología Educativa (en línea), n. 7, España, 1997, en: [http: / / nti.uji.es/docs/nti/Jordi_Adell_EDUTEC.html]. Consulta: 31 de julio de 2007.

BAUMAN, Zygmunt, Los retos de la educación en la modernidad líquida, Gedisa Barcelona, 2007.

BERGER, Peter y LUCKMAN, Thomas, La construcción social de la realidad, Amorrortu, Buenos Aires, 1997.

CABRERA, Valeria, «Democratización de la información en Chile. El lugar de la apropiación social de las TIC en la agenda digital 2004-2006», Comunicación y medios (en línea), n. 17, 2006, en: [http:/ / www.icei.uchile.cl / comunicacionymedios / 17.html]. Consulta: 2 de diciembre de 2008. 
CARRIÓN, Carmen (coord.), Educación para una sociedad del conocimiento, Comité Regional Norte de Cooperación con la UNESCO (en línea), México, 2007, en: [http://www.comitenorte.org.mx/libros/educacionsociedad.pdf]. Consulta: 22 de noviembre de 2009.

CASAS, Rosalba y DETTMER, Jorge, «Sociedad del conocimiento, capital intelectual y organizaciones innovadoras», en Giovanna Valenti; Mónica Casalet y Avaro Dante (coord.), Instituciones, sociedad del conocimiento y mundo del trabajo, Flacso, Plaza y Valdés, México, 2008, p. 21-53.

CASTELLS, Manuel, La era de la información. Economía, sociedad y cultura. La sociedad red, vol. I, Siglo XXI, Madrid, 2008.

CERVANTES, Edilberto, La Sociedad del Conocimiento. Oportunidades y estrategias, Comité Regional Norte de Cooperación con la UNESCO, Diálogos (en línea), México, 2006, en: [http:/ / www.comitenorte.org.mx/libros / sociedad_del_conocimiento.pdf]. Consulta 22 de noviembre de 2009.

CMSI, «Cumbre Mundial de la Sociedad de la Información». Declaración de principios y plan de acción de la CMSI (en línea), 2005, en: [www.itu.int/wsis]. Consulta 20 de enero de 2009.

$\mathrm{COBO}$, Cristóbal, Organización de la información y su impacto en la usabilidad de las tecnologías interactivas, Tesis Doctoral, México, 2005.

Aprendizaje adaptable y apropiación tecnológica: Reflexiones prospectivas, 2007, en: [http:// desinuamc.org/autoestudio3/ponencias/ponencia33.pdf]. Consulta: 24 de julio de 2009.

DEL ÁLAMO, Óscar, «Esperanza tecnológica: Internet para los pueblos indígenas de América Latina», Revista Futuros (en línea), vol. II, n. 6, 2004, en: [http: / / www.revistafuturos.info/futuros_6/internet_ind_1.htm]. Consulta: 23 de julio de 2009.

DUCOING, Patricia y MIRANDA, Francisco, «Necesidades de formación profesional y prácticas institucionales del docente en educación secundaria», Subsecretaría de Educación Básica y Normal, 
Dirección General de Investigación Educativa (en línea), Informes finales de investigación educativa, 2004, en:[http: / / www.ocyt.org.co / esocite/Ponencias_ESOCITEPDF/1MEX059.pdf]. Consulta: 30 de junio de 2009.

FLORIANÓPOLIS, Declaración de Florianópolis, en: [http: / / aplicaciones.conexionciudad.com/backend/imagenes/coloquio/docs /Declaracion_de_Florianopolis.pdf]. Consulta: 30 de marzo de 2010.

GÓMEZ MONT, Carmen, «El conocimiento indígena en México: Usos y prácticas cotidianas ante las tecnologías digitales», Sociedad del conocimiento y diversidad cultural (en línea), 2006 en: [http:/ / www.sociedadconocimiento.unam.mx/El_conocimiento_indigena_en_Mexico.pdf]. Consulta: 27 de abril de 2009.

MEDELLÍN, Sofía y HUERTA, V.E., «La promoción de las TIC para el desarrollo y los pueblos indígenas: ¿extensión o comunicación?», Community Informatics in Latin America and the Caribbean, 2006, en: [http:// cijournal.net/index.php/ciej/issue/view/18]. Consulta: 20 de enero de 2009.

MONTUSHI, Luisa, «La economía basada en el conocimiento: Importancia del conocimiento tácito y del conocimiento codificado», UCEMA. DT (en línea), n. 204, 2001, en: [www.cema.edu.ar/publicaciones/download/documentos/204.pdf]. Consulta: 7 de agosto de 2009.

MORALES, S.; MONJE, D. y LOYOLA, M., «Apropiación crítica de NTC'S como camino de inclusión en la Sociedad de la Información: el caso de los jóvenes de una escuela secundaria de la ciudad de Córdoba», Razón y palabra (en línea), vol. 52, 2004, en: [http: / / www.razonypalabra.org.mx/anteriores / n52/30MoralesM onjeyLoyola.pdf]. Consulta: 2 de diciembre de 2008.

SAGÁSTEGUI, Diana, «Usos y apropiaciones del Programa Enciclomedia en las escuelas primarias de Jalisco», COMIE (en línea), 2006, en: [http://www.comie.org.mx/congreso/memoria/v9/ponencias/at07/PRE1189534811.pdf]. Consulta: 16 de junio de 2008. 
SÁNCHEZ, Rolando, «La observación participante como escenario y configuración de la diversidad de significados», en María Luisa Tarrés (coord.), Observar, escuchar y comprender. Sobre la tradición cualitativa en la investigación social, COLMEX-FLACSOPORRÚA, México, 2008, p. 97-131.

SANDOVAL, Eduardo y MOTA, D., Indígenas y democracia en las Tecnologías de la Información y la Comunicación (en línea), 2005, en: [http:/ / www.ocyt.org.co/esocite/Ponencias_ESOCITEPDF/ 1MEX059.pdf]. Consulta: 20 de enero de 2009.

TREJO, Raúl, «Vivir en la sociedad de la información. Orden global y dimensiones locales en el universo digital», CTS+I. OEI (en línea), vol. 1, 2001, en: [http://www.oei.es/revistactsi / numero1/trejo.htm]. Consulta 9 de diciembre de 2009.

UNESCO, Hacia las sociedades del conocimiento, Informe mundial de la Organización de las Naciones Unidas para la Educación, la Ciencia y la Cultura, 2005. 